\title{
Polyinosinic-polycytidylic Acid/Inactivated Rabies Virus Vaccine YS-ON-001
}

\author{
National Cancer Institute
}

\section{Source}

National Cancer Institute. Polyinosinic-polycytidylic Acid/Inactivated Rabies Virus Vaccine

YS-ON-001. NCI Thesaurus. Code C148534.

A cancer vaccine composed of inactivated rabies vaccine (RV-V) and polyinosinicpolycytidylic acid (poly IC; poly I-C), an immunostimulant and Toll-like receptor 3 (TLR3) agonist, with potential immunostimulating and antineoplastic activities. Upon administration of the poly IC/inactivated RV-V YS-ON-001, the RV-V stimulates the immune system in various ways, such as promoting the activation and proliferation of $T$ helper type 1 (Th1) cells, dendritic cells (DCs), B cells and natural killer cells (NKs), promoting macrophage M1 polarization, downregulating regulatory $\mathrm{T}$-cells (T regs), and inducing the production of anti-tumor cytokines. This re-activates immune responses in the suppressed tumor microenvironment (TME), leading to an induction of cytotoxic Tlymphocyte (CTL)-mediated immune response against the tumor cells, which cause tumor cell apoptosis and a reduction of tumor cell proliferation. Poly IC, a synthetic double-stranded RNA with one strand composed of a inosinic acid polymer and the other strand a cytidylic acid polymer, binds to TLR3 and stimulates the release of cytotoxic cytokines, such as interferon-gamma (IFNg). This may boost the immune response against the tumor cells. 